\title{
Broadband Miniaturized Bandpass Filter with Circular Stubs for Compact Wireless and Mobile Communication Systems
}

\author{
Yulan Zhang', Heather Song ${ }^{2}$ \\ ${ }^{1}$ Intel Co., Hillsboro, USA; ${ }^{2}$ Department of Electrical and Computer Engineering, University of Colorado, Colorado Springs, USA. \\ Email: hsong@uccs.edu
}

Received December $20^{\text {th }}, 2012$; revised January $19^{\text {th }}, 2013$; accepted February $5^{\text {th }}, 2013$

\begin{abstract}
A miniaturized square resonator bandpass filter with circular stubs was designed, fabricated, and characterized. Analytical calculations were carried out to determine the critical filter parameters and the design was optimized using a 3D electromagnetic finite-element solver. The measured results were in good agreement with the designed results. The proposed filter exhibits significant improvement in bandwidth compared to the conventional square resonator bandpass filters.
\end{abstract}

Keywords: Miniaturized Square Resonator; Bandpass Filter; Circular Stub; Electromagnetic Simulation

\section{Introduction}

Size reduction is an important issue in developing high performance miniature RF filters in wireless and mobile communication systems. The conventional edge parallelcoupled filter and hairpin filter are constructed in identical resonators cascaded in alternating series orientation which show steeper roll-off on the lower frequency side than on the higher frequency side [1-5]. Research on the modified miniaturized parallel-coupled filters has been reported to improve the filter's symmetric response and upper stopband rejection characteristics [6-11]. In this paper, a new microstrip resonator filter with circular stubs is investigated for miniaturization and improvement in bandwidth. It is also known that the proposed filter geometries provide good upper stopband rejection characteristics. Analytical calculations and 3D electromagnetic simulations were carried out to determine the filter characteristics. Based on the design, a four-pole Chebyshev microstrip bandpass filter with circular stubs was fabricated and compared with design results.

\section{Design of a Miniaturized Bandpass Filter with Circular Stubs}

Miniaturization of filters can be explained as shown in Figure 1. It has been demonstrated that the conventional hairpin filters in Figure 1(a) can be miniaturized by a pair of coupled lines folded inside the resonator as shown in Figure 1(b). By utilizing the proposed filter in this work shown in Figure 1(c), one can achieve further miniaturization. This is due to the fact that the capacitance provided by the circular stubs decrease the resonant frequency. Figure 2 shows a typical resonator structure of the circular stub loaded bandpass filter. The resonator consists of a square ring with a pair of coupled lines folded inside the resonator which is connected to circular stubs. Compared to the conventional hairpin filters, the capacitance between both ends of the resonator allows miniaturization of the filter. The resonant frequency is reduced due to the shunt capacitance of the circular stub. Theoretical analysis on the radial stub loaded microstrip was reported [12-14]. The proposed structure can be analyzed as a capacitively loaded transmission line [15]. Figure 3 shows an equivalent circuit model for the proposed structure. A $C_{x}$ is the total capacitance which

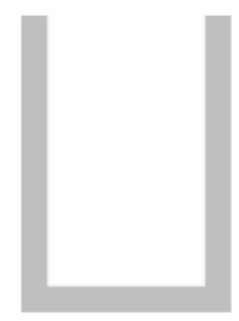

(a)

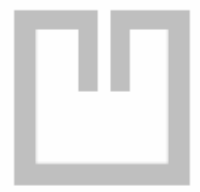

(b)

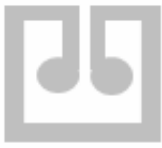

(c)
Figure 1. Miniaturization of resonator filters: (a) Conventional hairpin resonator; (b) Square resonator with folded coupled line; and (c) Miniaturaized filter loaded with circular stubs. 


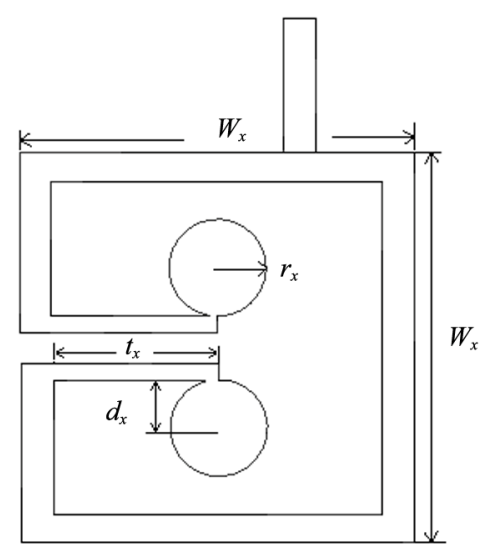

Figure 2. Schematic of the miniaturized bandpass filter with circular stubs.

includes the circular stub mode capacitance of the foldedcoupled lines, and a fringing capacitance between openend circular stub and the adjacent microstrip line. The characteristic impedance, the propagation constant, and the resonator width are represented by $Z_{l}, \beta_{l}$, and $W_{x}$, respectively. The resonant frequencies of the equ- ivalent circuit can be obtained by applying the boundary conditions $I_{1}=I_{2}=0$ for a nontrivial solution of $V_{1}$ and $V_{2}$. The resonant frequency can be obtained from [15, 16]

$$
\tan \left(\frac{\theta_{e}}{2}\right)=\left(\frac{1}{\pi f_{c} C_{x} Z_{l}}\right)
$$

where $f_{c}$ is the center frequency, $Z_{l}$ is the characteristic impedance of the stub, and $\theta_{e}\left(=\beta_{l} W_{x}\right)$ is the electrical length.

Bandpass filters can be defined by three characteristics: resonator structure, coupling coefficients which are the coupling between resonators, and external quality factor which is the coupling to the terminations [17]. A four pole Chebyshev $0.01 \mathrm{~dB}$ bandpass filter was designed on a RT/Duroid substrate with a thickness of $0.635 \mathrm{~mm}$ and a relative dielectric constant of 10.2. For the center frequency of $1.56 \mathrm{GHz}$, bandwidth of $13 \%$ and an external quality factor, $Q_{e}$ of 5.5 were obtained. The coupling coefficients were $K_{12}=0.1407, K_{23}=0.1034, K_{34}=$ 0.1407 .

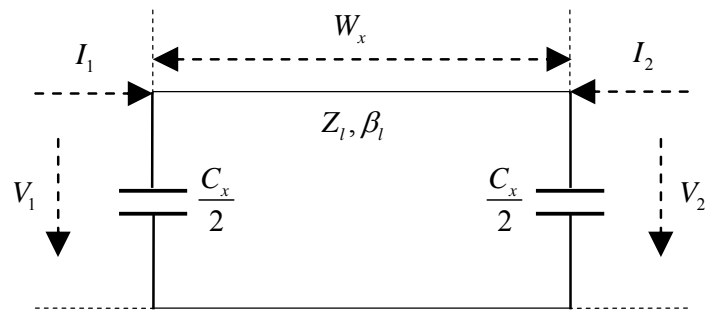

Figure 3. Equivalent circuit model for the miniaturized bandpass filter with circular stubs.
The fundamental resonance frequency depends on the dimensions of the connecting stub length and width, position of the circular stub connecting area, and number of stubs. Extensive 3D electromagnetic wave simulations were carried out using a 3D EM solver [18] in order to obtain the optimized parameters for the miniaturized bandpass filter with circular stubs. The optimized parameters are shown in Table 1. Figure 4 shows the filter simulated response for various values of the circular stub radius $r_{x}$, the distance from the circular stub center to the line $d_{x}$, and the circular position of the stub $t_{x}$. The optimized structure which yields the widest bandwidth with steep filter roll-off characteristics shows $W_{x}$ of 7.4 $\mathrm{mm}, r_{x}$ of $1.00 \mathrm{~mm}, d_{x}$ of $1.20 \mathrm{~mm}$, and $t_{x}$ of 3.10 $\mathrm{mm}$. Figure 5 which shows external quality factor, $Q_{e}$ for various values of $r_{x}, d_{x}$, and $t_{x}$. The optimized dimensions of $r_{x}$ of $1.00 \mathrm{~mm}, d_{x}$ of $1.20 \mathrm{~mm}$, and $t_{x}$ of $3.10 \mathrm{~mm}$ are indicated as minimum points in Figure 5 which implies that these dimensions provide the widest bandwidth.

\section{Measurement Results and Discussion}

A miniaturized bandpass filter with circular stubs was fabricated in the form of four identical resonators cascaded in alternating series orientation as shown in Figure 6. The substrate used was RT/d6010LM of Rogers Corporation. The $S_{21}$ measurement results are compared in Figure 7 along with the simulated and the modified simulated results. The modified simulation curve was obtained using the actual fabricated circuit dimensions, dielectric constant, and conductor material. The measured passband loss was $-3.4 \mathrm{~dB}$ at the midband which is caused by the dielectric loss of the substrate and conductor loss of the copper. The accuracy limitation in circuit fabrication using LPKF is believed to be the contributing factor for the bandwidth difference between simulated and measured results. Figure 8 shows the measured, simulated, and modified simulated results for $S_{11}$. We have observed that as the width of the trace of the filter increases, the filter operating frequency increases, ripple decreases, and bandwidth increases. Compared to previously reported work on capacitively loaded parallel coupled bandpass filter [7,10,16-18], the proposed

Table 1. Optimized design parameters for the miniaturized bandpass filter with circular stubs.

\begin{tabular}{|c|c|}
\hline Filter Parameters & Value \\
\hline$W_{x} \quad(\mathrm{~mm})$ & 7.40 \\
\hline$r_{x}(\mathrm{~mm})$ & 1.00 \\
\hline$d_{x}(\mathrm{~mm})$ & 1.20 \\
\hline$t_{x} \quad(\mathrm{~mm})$ & 3.10 \\
\hline$f_{c}(\mathrm{GHz})$ & 1.56 \\
\hline
\end{tabular}




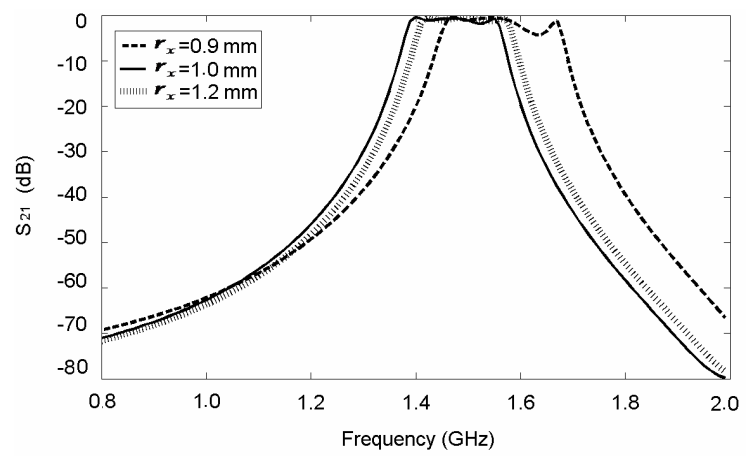

(a)

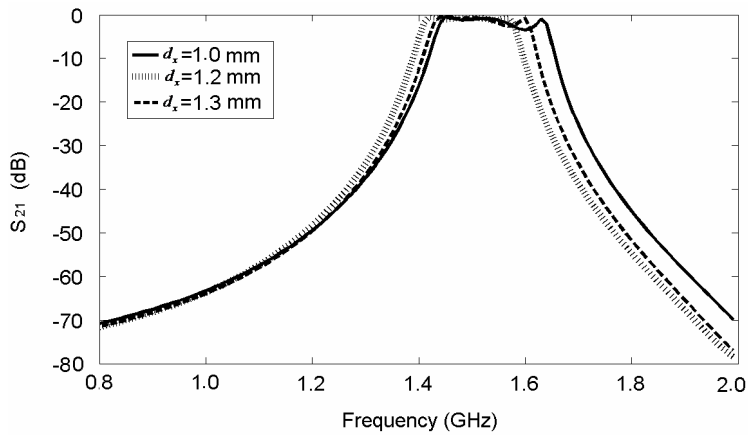

(b)

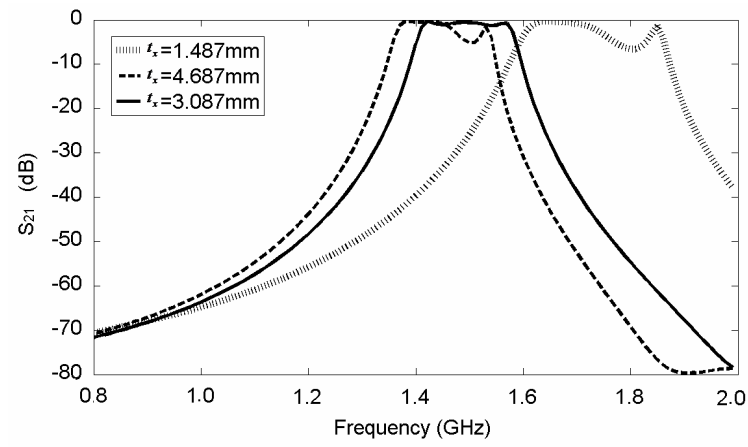

(c)

Figure 4. Simulated $S_{21}$ response of the four-pole miniaturized bandpass filter with circular stubs for various values of (a) $r_{x}$, (b) $d_{x}$, and (c) $t_{x}$.

filter provides $160 \%$ bandwidth improvement.

\section{Conclusion}

A square resonator bandpass filter with circular stubs is designed, fabricated, and characterized for miniaturization and bandwidth improvement. Based on the analytical calculations and 3D electromagnetic simulation, a four-pole Chebyshev microstrip bandpass filter was constructed with the substrate of RT/d6010LM from Rogers Corporation and was fabricated using LPKF Protomat machine. The measurement result was compared with the simulated and modified simulated results. The modified simulated curve was adjusted to the actual sizes of the

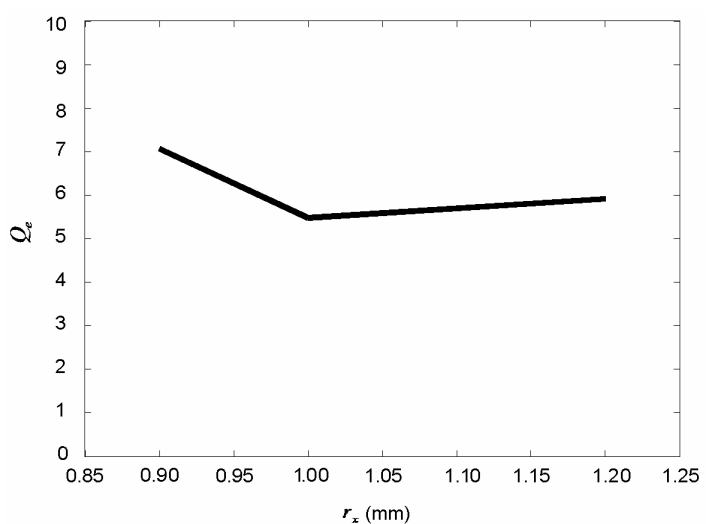

(a)

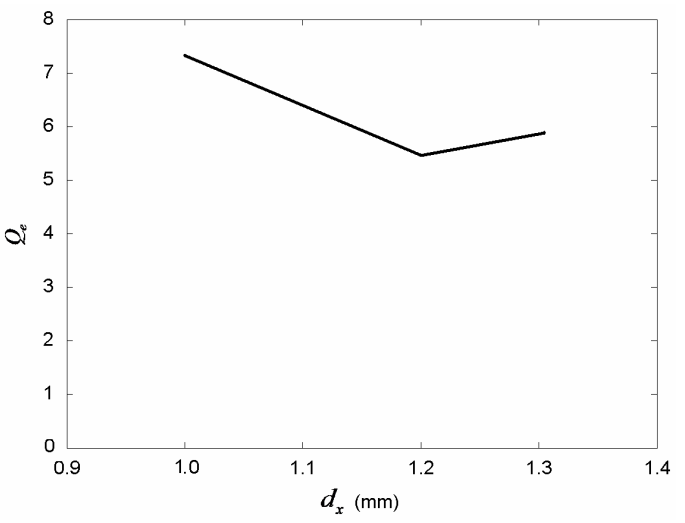

(b)

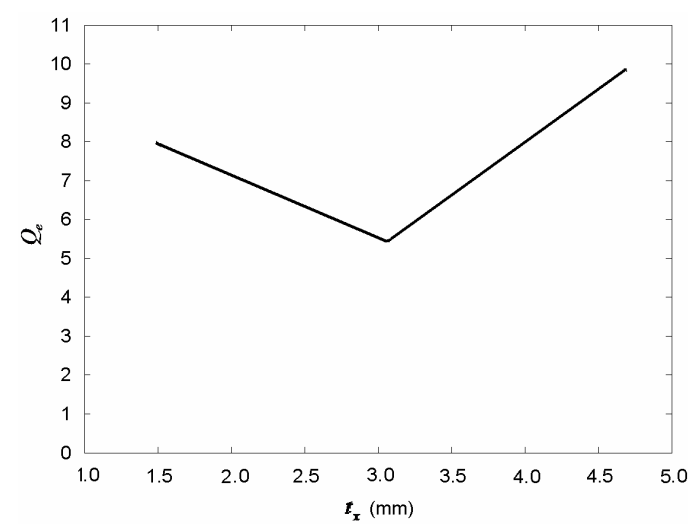

(c)

Figure 5. Simulated external quality factor, $Q_{e}$ of the miniaturized bandpass filter with circular stubs for various values of (a) $r_{x}$, (b) $d_{x}$, and (c) $t_{x}$.

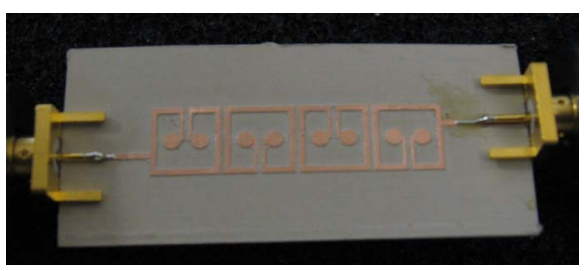

Figure 6. Photo of the fabricated four pole miniaturized bandpass filter with circular stubs. 


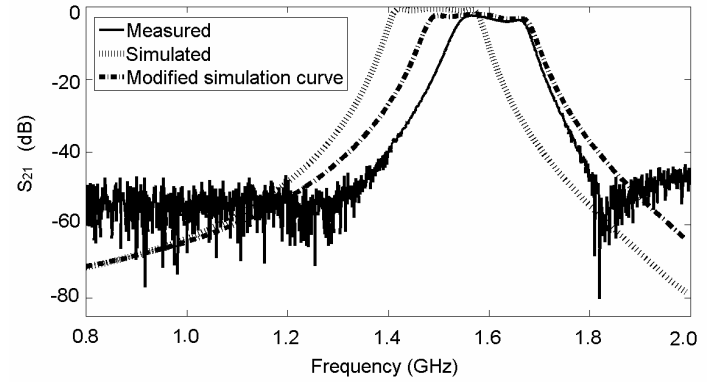

Figure 7. Comparison of simulated and measured $S_{21}$ for the optimized miniaturized bandpass filter with circular stubs.

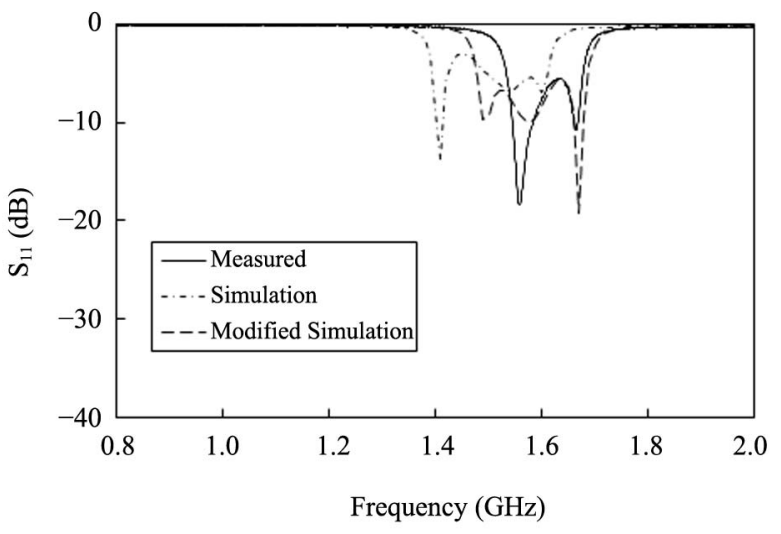

Figure 8. Comparison of simulated and measured $S_{11}$ for the optimized miniaturized bandpass filter with circular stubs.

fabricated circuit, dielectric constant, and conductor material. The measured results were in good agreement with the designed results. The proposed filter is miniaturized in size compared to the conventional hairpin resonator and square resonator with folded coupled lines. Furthermore, it exhibits significant improvement in bandwidth compared to the conventional square resonator bandpass filters. The miniaturized bandpass filter with circular stubs can be potentially used for compact wireless and mobile communication systems.

\section{Acknowledgements}

The authors would like to thank Dr. Mark Wickert for his advice on circuit fabrication. We would also like to thank Tom Mulcahy and Dr. Jim Wigle for their technical support on LPKF Protomat.

\section{REFERENCES}

[1] M. Sagawa, L. Takahashi and M. Makimoto, "Miniaturized Hairpin Resonator Filters and Their Application to Receiver Front-End MICs," IEEE Transactions on Microwave Theory Techniques, Vol. 37, No. 12, 1989, pp.
1991-1997. doi:10.1109/22.44113

[2] J. S. Wong, "Microstrip Tapped-Line Filter Design," IEEE Transactions on Microwave Theory Techniques, Vol. 27, No. 1, 1979, pp. 44-50. doi:10.1109/TMTT.1979.1129556

[3] E. G. Cristal and S. Frankel, "Hairpin-Line and Hybrid Hairpin Line/Half-Wave Parallel Coupled Line Filters," IEEE Transactions on Microwave Theory Techniques, Vol. 20, No. 11, 1972, pp. 719-728. doi:10.1109/TMTT.1972.1127860

[4] Y. Di, P. Gardner, P. S. Hall, H. Ghafouri-Shiraz and J. Zhou, "Multiple-Coupled Microstrip Hairpin-Resonator Filter," IEEE Microwave and Wireless Components Letters, Vol. 13, No. 12, 2003, pp. 532-534. doi:10.1109/LMWC.2003.819377

[5] A. Hasan and A. E. Nadeem, "Novel Microstrip Hairpin Narrowband Bandpass Filter Using via Ground Holes," Progress in Electromagnetics Research, Vol. 78, 2008, pp. 393-419. doi:10.2528/PIER07091401

[6] M. Makimoto and S. Yamshita, "Bandpass Filters Using Parallel Coupled Stripline Stepped Impedance Resonators," IEEE Transactions on Microwave Theory Techniques, Vol. 28, No. 12, 1980, pp. 1413-1417. doi:10.1109/TMTT.1980.1130258

[7] J. S. Hong and M. J. Lancaster, "End-Coupled Microstrip Slow-Wave Resonator Filters," Electronics Letters, Vol. 32, No. 16, 1996, pp. 1494-1496. doi:10.1049/el:19960959

[8] T. Itoh and C. Y. Chang, "A Modified Parallel-Coupled Filter Structure Improves the Upper Stop-Band Performance and Response Symmetry," IEEE Transactions on Microwave Theory Techniques, Vol. 39, No. 2, 1991, pp. 310-314. doi:10.1109/22.102975

[9] J. S. Hong and M. J. Lancaster, "Microstrip Slow-Wave Open-Loop Resonator Filters," Proceedings of the International Microwave Symposium IEEE MTT-S, Denver, 8-13 June 1997, pp. 713-716. doi:10.1109/MWSYM.1997.602890

[10] L. Zhu and K. Wu, "Accurate Circuit Model of Interdigital Capacitor and Its Application to Design of New QuasiLumped Miniaturized Filters with Suppression of Harmonic Resonance," IEEE Transactions on Microwave Theory Techniques, Vol. 48, No. 3, 2000, pp. 347-356. doi: $10.1109 / 22.826833$

[11] S. Y. Lee and C. M. Tsai, "A New Network Model of Miniaturized Hairpin Resonators and Its Applications," Proceedings of the International Microwave Symposium IEEE MTT-S, Boston, 11-16 June 2000, pp. 1161-1164. doi:10.1109/MWSYM.2000.863564

[12] H. A. Atwater, "Microstrip Reactive Circuit Elements," IEEE Transactions on Microwave Theory Techniques, Vol. 31, No. 6, 1983, pp. 488-491. doi:10.1109/TMTT.1983.1131529

[13] F. Giannini, R. Sorrentino and J. Vrba, "Planar Circuit Analysis of Microstrip Radial Stub," IEEE Transactions on Microwave Theory Techniques, Vol. 32, No. 12, 1984, pp. 1652-1655. doi:10.1109/TMTT.1984.1132907

[14] F. Giannini, M. Ruggieri and J. Vrba, "Shunt-Connected 
Microstrip Radial Stubs," IEEE Transactions on Microwave Theory Techniques, Vol. 34, No. 3, 1986, pp. 363366. doi:10.1109/TMTT.1986.1133347

[15] J. S. Hong and M. J. Lancaster, "Microstrip Filters for RF/Microwave Applications," John Wiley \& Sons, Inc., New York, 2001. doi:10.1002/0471221619

[16] J. S. Hong and M. J. Lancaster, "Theory and Experiment of Novel Microstrip Slow-Wave Open-Loop Resonators
Filters," IEEE Transactions on Microwave Theory Techniques, Vol. 45, No. 12, 1997, pp. 2358-3465. doi:10.1109/22.643844

[17] G. Mattaei, L. Young and E. M. T. Jones, "Microwave Filters, Impedance-Matching Networks, and Coupling Structures," Artech House, Norwood, 1980.

[18] ANSYS HFSS Software.

http://www.ansoft.com/products/hf/hfss/ 